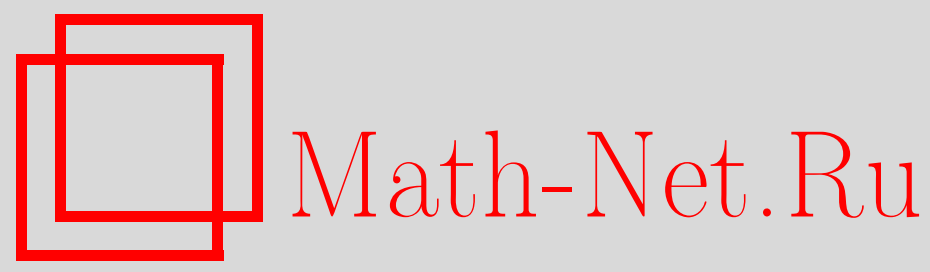

Д. В. Захарова, Симметрические линейные пространства графов, Дискрет. матем., 2011, том 23, выпуск 2, 103-107

DOI: https://doi.org/10.4213/dm1145

Использование Общероссийского математического портала Math-Net.Ru подразумевает, что вы прочитали и согласны с пользовательским соглашением http://www.mathnet.ru/rus/agreement

Параметры загрузки:

IP: 35.173 .137 .237

26 апреля 2023 г., 18:11:51 
УДК 519.1

\title{
Симметрические линейные пространства графов
}

\author{
() 2011 г. Д. В. Захарова
}

\begin{abstract}
Рассматриваются множества графов, замкнутые относительно симметрической разности и переименования вершин. Доказывается, что при любом $n$ имеется не более 14 таких множеств, состоящих из графов с $n$ вершинами.
\end{abstract}

\section{1. Введение}

В статье продолжается начатое в [1] исследование симметрических пространств графов. Классом графов называется множество обыкновенных графов, замкнутое относительно переименования вершин. Если $\mathbf{X}-$ класс графов, то через $\mathbf{X}_{n}$ обозначается множество всех графов из $\mathbf{X}$ с множеством вершин $\{1,2, \ldots, n\}$. Сумма по модулю 2 графов $G_{1}=\left(V_{1}, E_{1}\right)$ и $G_{2}=\left(V_{2}, E_{2}\right)$ есть граф

$$
G 1 \oplus G 2=\left(V_{1} \cup V_{2},\left(E_{1}-E_{2}\right) \cup\left(E_{2}-E_{1}\right)\right) \text {. }
$$

Если $V_{1}=V_{2}$, то граф $G_{1} \oplus G_{2}$ будем называть симметрической разностью графов $G_{1}$ и $G_{2}$.

В [1] рассматривались классы графов, замкнутые относительно сложения по модулю 2 и удаления изолированных вершин, и доказано, что существует ровно 5 таких классов. Очевидно, имеется бесконечно много классов, замкнутых только относительно сложения по модулю 2 (например, при любом $k$ множество всех графов с не менее чем $k$ вершинами). Если $\mathbf{X}$ - такой класс графов, то множество $\mathbf{X}_{n}$ при любом $n$ замкнуто относительно симметрической разности. Такое множество, то есть множество графов с множеством вершин $\{1,2, \ldots, n\}$, замкнутое относительно симметрической разности, будем называть симметрическим линейным пространством графов (СЛПГ) порядка $n$. В настоящей статье будет доказано, что при любом $n$ существует не более 14 СЛПГ порядка $n$. Перечислим эти множества (индекс $n$ опускаем):

В1 - все графы;

В2 - графы с четным числом ребер;

B3 - графы, у которых степени всех вершин имеют одинаковую четность;

B4 - графы, у которых степени вершин имеют одинаковую четность, совпадающую с четностью числа ребер;

В5 - графы, у которых степени всех вершин четны; 
$\mathbf{B 6}=\mathbf{B 2} \cap \mathbf{B 3} ;$

$\mathbf{B} 7=\mathbf{B} 2 \cap \mathbf{B 5} ;$

$\mathbf{L 1}$ - полные двудольные и полные кодвудольные (дополнительные к полным двудольным) графы;

$\mathbf{L 2}=\mathbf{L 1} \cap \mathbf{B 5}$ - полные двудольные и полные кодвудольные графы с четными степенями всех вершин;

$\mathbf{L 3}$ - полные двудольные графы с четными степенями и полные кодвудольные с нечетными степенями всех вершин;

L4 - полные двудольные графы;

$\mathbf{L 5}=\mathbf{L 4} \cap \mathbf{B 5}$ - полные двудольные графы с четными степенями всех вершин;

L6 - полный и пустой графы;

L7 - пустой граф.

Замкнутость всех этих множеств относительно симметрической разности легко проверяется.

Отметим, что при нечетном $n$ имеется не более 8 СЛПГ, так как в этом случае $\mathbf{B 3}=\mathbf{B 5}$, $\mathbf{B 4}=\mathbf{B 6}=\mathbf{B} 7, \mathbf{L 2}=\mathbf{L 3}=\mathbf{L 5}=\varnothing$.

На протяжении статьи число вершин $n$ считается фиксированным и рассматриваются графы с множеством вершин $\{1,2, \ldots, n\}$. При этом в обозначении множеств графов индекс, указывающий число вершин, будем опускать. Например, вместо $\mathbf{B} \mathbf{1}_{n}$ пишем просто В1.

Некоторые стандартные обозначения используются в статье не совсем обычным образом. Именно, $P_{k}$ и $C_{k}, k \leqslant n$, обозначают графы с $n$ вершинами, у которых вершины $1,2, \ldots, k$ порождают соответственно путь или цикл (с ребрами $(1,2),(2,3)$ и т.д.), остальные вершины - изолированные; $K_{p, q}-$ полный двудольный граф, в котором одна доля образована вершинами $1,2, \ldots, p$, другая образована вершинами $p+1, p+2, \ldots, p+q$; $\bar{G}-$ граф, дополнительный к графу $G$.

Если $G$ - граф, $f$ - подстановка на множестве его вершин, то через $G^{f}$ обозначаем граф, полученный из $G$ переименованием вершин в соответствии с $f$, то есть вершины $a$ и $b$ смежны в графе $G^{f}$ тогда и только тогда, когда вершины $f^{-1}(a)$ и $f^{-1}(b)$ смежны в графе $G$. Подстановку записываем в виде произведения циклов, опуская неподвижные вершины.

Для графа $G$ через $[G]$ обозначаем минимальное СЛПГ, содержащее этот граф.

\section{2. Классы L1-L7}

Классы L1-L7 являются подмножествами класса L1. Последний класс - наследственный, то есть замкнут относительно удаления вершин. Поэтому он может быть охарактеризован запрещенными графами - минимальными по отношению “быть порожденным подграфом” графами, не содержащимися в нем. Покажем, что множество минимальных запрещенных подграфов для $\mathbf{L} \mathbf{1}$ состоит из пяти четырехвершинных графов. Пусть $A_{1}$, $A_{2}, A_{3}$ - графы с множеством вершин $\{1,2,3,4\}$ и множествами ребер соответственно $\{(1,2)\},\{(1,2),(2,3)\},\{(1,2),(2,3),(3,4)\}$. Очевидно, что эти графы, а также графы $\bar{A}_{1}$ и $\bar{A}_{2}$, не принадлежат классу $\mathbf{L 1}$. 
Лемма 1. Если в графе $G$ нет порожденных подграфов, изоморфных графам $A_{1}, A_{2}, A_{3}$, $\bar{A}_{1}, \overline{A_{2}}, m o G \in \mathbf{L} \mathbf{1}$.

Доказательство. Граф $A_{3}$ - это $P_{4}$ в стандартных обозначениях. Известно [2], что граф, не содержащий $P_{4}$ в качестве порожденного подграфа, либо сам несвязен, либо несвязен дополнительный к нему. Если $G$ несвязен и не является пустым графом, то в нем имеется не более двух компонент связности (иначе существовал бы порожденный подграф $A_{1}$ ). Так как не существует порожденного подграфа $A_{2}$, каждая компонента есть полный подграф. Значит, $G-$ полный кодвудольный граф. Аналогично убеждаемся, что если $\bar{G}$ несвязен и не является полным графом, то $G$ - полный двудольный граф.

Следующая лемма позволяет в дальнейшем отдельно рассматривать классы B1-B7 и L1-L7.

Лемма 2. Если $\mathbf{X}-$ СЛПГ, то $\mathbf{X} \subseteq \mathbf{L 1}$ или $C_{4} \in \mathbf{X}$.

Доказательство. Допустим, что $\mathbf{X} \nsubseteq \mathbf{L} \mathbf{1}$ и $G \in \mathbf{X}-\mathbf{L 1}$. Тогда $G$ содержит порожденный подграф $H$, изоморфный одному из пяти запрещенных графов для L1. Допустим, что такой подграф порождается вершинами $1,2,3,4$. Покажем, что в любом случае $C_{4} \in[G]$.

Если $H$ изоморфен $A_{1}$ и содержит ребро $(1,2)$, то

$$
G \oplus G^{(1,3)} \oplus G^{(2,4)} \oplus G^{(1,4)(2,3)} \cong C_{4} .
$$

Если $H$ изоморфен $A_{2}$ и содержит ребра $(1,2),(2,3)$, то

$$
G \oplus G^{(1,2)} \oplus G^{(3,4)} \oplus G^{(1,2)(3,4)} \cong C_{4} .
$$

Если $H$ изоморфен $A_{3}$ и содержит ребра $(1,2),(2,3),(3,4)$, то

$$
G \oplus G^{(1,2)} \oplus G^{(3,4)} \oplus G^{(1,2)(3,4)} \cong C_{4} .
$$

В оставшихся двух случаях утверждение следует из предыдущих ввиду тождества

$$
\bar{G}_{1} \oplus \bar{G}_{2}=G_{1} \oplus G_{2} .
$$

Лемма 3. Если $\mathbf{X}-$ СЛПГ порядка $n, \mathbf{X} \subseteq \mathbf{L 1}, \mathbf{X} \neq \mathbf{L 6}$ u $\mathbf{X} \neq \mathbf{L} 7$, mo $K_{2 s, n-2 s} \in \mathbf{X}$ при любом $s \leqslant\lfloor n / 2\rfloor$.

Доказательство. Пусть $G$ - граф из $\mathbf{X}$, не являющийся ни полным, ни пустым, то есть $G$ - полный двудольный или полный кодвудольный граф с непустыми долями. Возьмем вершины $a$ и $b$ из разных долей. Тогда

$$
G \oplus G^{(a, b)} \cong K_{2, n-2}
$$

Пусть теперь $H=K_{2, n-2}$. Тогда

$$
H \oplus H^{(1,3)(2,4)} \oplus H^{(1,5)(2,6)} \oplus \ldots \oplus H^{(1,2 s-1)(2,2 s)}=K_{2 s, n-2 s} .
$$

Теорема 1. Если $\mathbf{X}-$ СЛПГ порядка п и $\mathbf{X} \subseteq \mathbf{L 1}$, то $\mathbf{X}$ совпадает с одним из множеств L1-L7.

Доказательство. Пусть $\mathbf{X} \subseteq \mathbf{L 1}, \mathbf{X} \neq \mathbf{L 6}, \mathbf{X} \neq \mathbf{L 7}$, то есть $\mathbf{X}$ содержит хотя бы один граф, не являющийся ни полным, ни пустым. Возможны следующие варианты. 
(1) В X все графы - полные двудольные.

(a) Степени всех вершин в каждом графе из $\mathbf{X}$ четны (в этом случае $n$ четно). Из леммы 3 следует, что $\mathbf{X}=\mathbf{L 5}$.

(b) В некотором графе $G \in \mathbf{X}$ имеется вершина нечетной степени. Тогда этот граф изоморфен $K_{2 s+1, n-2 s-1}$ при некотором $s$. По лемме $3, K_{2 s, n-2 s} \in \mathbf{X}$. Следовательно, $\mathbf{X}$ содержит граф $K_{2 s+1, n-2 s-1} \oplus K_{2 s, n-2 s}$, изоморфный $K_{1, n-1}$. Пусть $H=K_{1, n-1}$. Тогда

$$
H \oplus H^{(1,2)} \oplus H^{(1,3)} \oplus \ldots \oplus H^{(1, t)}=K_{t, n-t} .
$$

Таким образом, $\mathbf{X}=\mathbf{L 4}$.

(2) В X есть полный кодвудольный граф.

(a) Во всех полных двудольных графах из $\mathbf{X}$ степени всех вершин четны. Тогда $n$ четно, а в каждом полном кодвудольном графе из $\mathbf{X}$ степени всех вершин имеют одинаковую четность.

i. В каждом полном кодвудольном графе из $\mathbf{X}$ степени всех вершин нечетны. Пусть $\bar{K}_{2 t, n-2 t} \in \mathbf{X}$. По лемме $3, K_{2 s, n-2 s} \in \mathbf{X}$ при любом $s$. Следовательно, в $\mathbf{X}$ содержится полный граф $K_{n}=\bar{K}_{2 t, n-2 t} \oplus K_{2 t, n-2 t}$ и граф $\bar{K}_{2 s, n-2 s}=K_{n} \oplus K_{2 s, n-2 s}$ при любом $s$, то есть, $\mathbf{X}=\mathbf{L 3}$.

ii. В каждом полном кодвудольном графе из $\mathbf{X}$ степени всех вершин четны. Пусть $\bar{K}_{2 t+1, n-2 t-1} \in \mathbf{X}$. Тогда $\mathbf{X}$ содержит граф $\bar{K}_{2 t+1, n-2 t-1} \oplus K_{2 t, n-2 t}$, изоморфный $\bar{K}_{1, n-1}$. Но $\bar{K}_{1, n-1} \oplus K_{2 s, n-2 s} \cong \bar{K}_{2 s-1, n-2 s-1}$. Следовательно, $\mathbf{X}=\mathbf{L} 2$.

iii. В $\mathbf{X}$ есть полные кодвудольные графы как с четными, так и с нечетными степенями. Допустим, $\mathbf{X}$ содержит графы $\bar{K}_{2 s, n-2 s}$ и $\bar{K}_{2 t+1, n-2 t-1}$. Тогда он содержит также граф $\bar{K}_{2 s, n-2 s} \oplus \bar{K}_{2 t+1, n-(2 t+1)} \cong K_{2 t+1-2 s, n-2 t-1+2 s)}$, то есть полный двудольный граф с нечетными степенями. Это противоречит условию 2 (а).

(b) В некотором графе $G \in \mathbf{X}$ имеется вершина нечетной степени. Как показано в 1 (b), в этом случае $\mathbf{X}$ содержит все полные двудольные графы. Кроме того, в нем имеется полный кодвудольный граф. Но тогда, рассуждая как в 2 (a), приходим к выводу, что $\mathbf{X}$ содержит все полные кодвудольные графы, то есть $\mathbf{X}=L 1$.

\section{3. Классы В1-В7}

Согласно лемме 2, любое СЛПГ, отличное от описываемых теоремой 1 , содержит граф $C_{4}$. Если $G-$ произвольный граф с $n$ вершинами, то из него и графов, изоморфных $C_{4}$, можно получить граф весьма специального вида.

Лемма 4. Для любого графа $G$ с $n$ вершинами существует граф $H \in\left[C_{4}\right]$ такой, что в графе $G \oplus H$ каждая компонента связности является звездой или треугольником, причем имеется не более одной компоненты-треугольника.

Доказательство. Допустим, что в графе $G$ имеется подграф, изоморфный $P_{4}$. Возьмем граф $F$, изоморфный $C_{4}$, в котором три ребра совпадают с ребрами этого графа $P_{4}$. В 
графе $G \oplus F$ ребер меньше, чем в графе $G$. Действуя таким образом, можно получить граф, в котором нет подграфов, изоморфных $P_{4}$. В таком графе каждая компонента связности является звездой или треугольником. Нетрудно видеть, что граф, состоящий из двух компонент-треугольников, раскладывается в симметрическую разность трех графов, изоморфных $C_{4}$.

Лемма 5. Справедливо равенство $\mathbf{B} 7=\left[C_{4}\right]$.

Доказательство. Включение $\left[C_{4}\right] \subseteq \mathbf{B 7}$ очевидно. Пусть $G \in \mathbf{B 7}, H-$ граф, существование которого утверждается в лемме 4 . В обоих графах $G$ и $C_{4}$ число ребер и все степени четны, поэтому то же будет верно и для графа $G \oplus H$. Значит, в нем не может быть компонент-звезд, состоящих более чем из одной вершины, не может быть и компонентытреугольника, то есть $G \oplus H-$ пустой граф. Следовательно, $G=H \in\left[C_{4}\right]$.

Теорема 2. Если $\mathbf{X}-$ СЛПГ и $\mathbf{X} \nsubseteq \mathbf{L 1}$, то $\mathbf{X}$ совпадает с одним из множеств $\mathbf{B 1 - B 7 .}$

Доказательство. Из лемм 3 и 5 следует, что любое СЛПГ, не являющееся подпространством пространства $\mathbf{L 1}$, содержит подпространство $\mathbf{B} 7=\left[C_{4}\right]$. Если при этом в $\mathbf{X}$ все графы имеют четное число ребер и четные степени всех вершин, то $\mathbf{X}=\mathbf{B} 7$. Рассмотрим другие возможные случаи.

1. Во всех графах из $\mathbf{X}$ степени всех вершин четны и имеется граф $G \in \mathbf{X}$ с нечетным числом ребер. Пусть $H$ - граф, существование которого утверждается в лемме 4 . Тогда $G \oplus H \cong C_{3}$. Любой цикл $C_{k}$ можно представить как симметрическую разность графов, изоморфных $C_{3}$ и $C_{4}$. Любой граф с четными степенями всех вершин является объединением циклов. Следовательно, $\mathbf{X}=\mathbf{B 5}$.

2. В Х имеется граф $G$, в котором есть вершины и с четными, и с нечетными степенями. Из него и графа $C_{4}$ с помощью симметрической разности можно получить граф $H$, в котором есть вершина $a$ степени 0 и вершина $b$ степени 1 . Тогда $H \oplus H^{(a, b)} \cong P_{3}$. Далее, $P_{3} \oplus P_{3}^{(1,2,3,4)} \cong 2 K_{2}$ (граф с двумя ребрами, не имеющими общей вершины). Очевидно, из $P_{3}$ и $2 K_{2}$ можно получить любой граф с четным числом ребер. Следовательно, если в $\mathbf{X}$ все графы имеют четное число ребер, то $\mathbf{X}=\mathbf{B 2}$. Если же в $\mathbf{X}$ есть граф с нечетным числом ребер, то из него и графов $P_{3}$ и $2 K_{2}$ можно получить граф с одним ребром, а из него - любой граф. Значит, в этом случае $\mathbf{X}=\mathbf{B} 1$.

3. В X имеются графы и с четными, и с нечетными степенями, но в каждом графе степени всех вершин имеют одинаковую четность. Графы с четными степенями образуют подпространство X0, а графы с нечетными - смежный класс X1. Как показано выше, X0 совпадает с одним из пространств В7, B5. Если $\mathbf{X 0}=\mathbf{B 5}$, то есть состоит из всех графов с четными степенями, то $\mathbf{X} \mathbf{1}$ состоит из всех графов с нечетными степенями и $\mathbf{X}=\mathbf{B 3}$.

Допустим, что $\mathbf{X 0}=\mathbf{B 7}$, то есть в $\mathbf{X 0}$ все графы имеют четное число ребер. Тогда в $\mathbf{X} 1$ либо у всех графов четное число ребер, либо у всех нечетное. В первом случае $\mathbf{X}=\mathbf{B 6}$, во втором $\mathbf{X}=\mathbf{B} 4$.

\section{Список литературы}

1. Алексеев В. Е., Захарова Д. В., О симметрических пространствах графов. Дискретный анализ $и$ исследование операций (2007) 14, №1, 21-26.

2. Corneil D. G., Lerchs H., Stewart-Burlingham L., Complement reducible graphs. Discrete Appl. Math. (1981) 13, 163-174.

Статья поступила 15.10.2008. 